\title{
Long-term In vivo Imaging and Measurement of Dendritic Shrinkage of Retinal Ganglion Cells
}

${ }^{1}$ Christopher Kai-shun Leung, MD, MB ChB, ${ }^{2}$ Robert N Weinreb, MD, ${ }^{1}$ Zhi wei Li, BM, ${ }^{1}$ Shu Liu BM, ${ }^{2}$ James

D Lindsey, PhD, ${ }^{1}$ Nathan Choi, BEng, ${ }^{1}$ Lan Liu, BM, ${ }^{1}$ Carol Yim-lui Cheung, PhD, ${ }^{1}$ Cong Ye BM,,${ }^{1,3}$ Kunliang Qiu, BM, ${ }^{1}$ Lijia Chen, BM, ${ }^{4}$ Wing-ho Yung, DPhil, ${ }^{5}$ Jonathan G Crowston, MD, ${ }^{6}$ Mingliang Pu, PhD, ${ }^{7}$ Kwok Fai So, PhD, ${ }^{1}$ Chi Pui Pang, DPhil, ${ }^{1}$ Dennis Shun-Chiu Lam, MD, FRCOphth

\section{Affiliation:}

1. Department of Ophthalmology and Visual Sciences, The Chinese University of Hong Kong, Hong Kong, P.R.China.

2. Hamilton Glaucoma Center, University of California, San Diego, CA, USA

3. Joint Shantou International Eye Center of Shantou University and Chinese University of Hong Kong, P.R.China.

4. School of Biomedical Science, The Chinese University of Hong Kong, Hong Kong, P.R.China.

5. Department of Ophthalmology, University of Melbourne, Australia.

6. Department of Anatomy and Embryology, School of Basic Medical Sciences, Peking University, P.R. China

7. State Key Laboratory of Brain and Cognitive Sciences, Department of Anatomy, University of Hong Kong, P.R. China

\section{Corresponding author/Address for reprints:}

Dr. Christopher Kai-shun Leung

Department of Ophthalmology \& Visual Sciences, The Chinese University of Hong Kong, Hong Kong Eye Hospital, 147K Argyle Street, Kowloon, Hong Kong

Tel: +85227623181

Fax: +85227159490

E-mail:tlims00@hotmail.com

This project was supported by a CUHK research grant and the Glaucoma Foundation.

The authors have no commercial relationship to disclose. 


\begin{abstract}
PURPOSE: To monitor and measure dendritic shrinkage of retinal ganglion cells (RGCs) in a strain of transgenic mice (Thy-1 YFP) that express yellow fluorescent proteins in neurons under the control of a Thy-1 promoter.

METHODS: A total of 125 RGCs from 16 eyes of the Thy-1 YFP transgenic mice were serially imaged with a confocal scanning laser ophthalmoscope for 6 months after optic nerve crush. With quantitative analysis of cell body area, axon diameter, dendritic field, number of terminal branches, total dendritic branch length, branching complexity, symmetry, distance from the optic disc, we characterized the morphology of RGCs, described the patterns of axonal and dendritic degeneration, identified the morphological predictors for cell survival, and estimated the rate of dendritic shrinkage.

RESULTS: RGC damage was observed prospectively to begin with progressive dendritic shrinkage, followed by loss of the axon and the cell body. In a small proportion of RGCs, progressive axonal changes with fragmentation, beading, retraction and bulb formation were also observed. RGCs with a larger dendritic field and a longer total dendritic branch length in general have a better survival probability. The rate of dendritic shrinkage was variable with a slower rate observed in cells having a larger dendritic field, a longer total dendritic branch length and being distant from the optic disc.

CONCLUSIONS: Estimating the probability of RGC survival and measuring the rate of dendritic shrinkage could become a new paradigm investigating neuronal degeneration and evaluating the response of neuroprotective treatment.
\end{abstract}


Progressive neuronal damage is a common feature of neurodegenerative diseases. While visualizing the morphology of individual neurons is pivotal to studying neuronal degeneration and neuroprotection, direct observation of the spatial and temporal changes of axonal and dendritic arborization has been difficult in the mammalian central nervous system. In vivo acute axonal degeneration was first demonstrated in the spinal cord with time-lapse imaging. ${ }^{1,2}$ However, this model does not work on concomitant visualization of dendritic trees and cell bodies. Because of the clear optical media of the eye, retinal ganglion cells (RGCs) represent a unique neuronal cell type that offers the opportunity for simultaneous evaluation of dendritic and axonal structures. Despite the fact that the RGCs can be visualized in vivo with injection of neuronal tracers to the superior colliculus, ${ }^{3,4}$ most neuronal tracers do not reveal the fine details of the dendritictrees. Short survival time and fading of neuronal tracers also impedes longitudinal evaluation of cell survival. ${ }^{5}$

The introduction of a strain of transgenic mice that express fluorescent protein in RGCs under the control of a neuron-specific fragment of the Thy-1 promoter offers a non-invasive method for longterm monitoring of progressive neuronal changes. ${ }^{6}$ We recently modified a confocal scanning laser ophthalmoscope (CSLO) to image RGCs in transgenic mice expressing cyan florescent protein under the control of the promoter for Thy-1 (Thy1-CFP23Jrs mice)..$^{7-9}$ Although individual RGC bodies can be identified, axon and dendritic arborization cannot be visualized in this animal strain in vivo. Another mouse strain that can be used for in vivo visualization of RGC is Thy1-YFP16Jrs. Because of strain-tostrain variation in transgene expression, ${ }^{6}$ less than $1 \%$ of RGCs are labeled. The relatively low density of YFP (yellow florescent protein) positive RGCs in the retina of these mice facilitates visualization and demarcation of dendritic arborization of individual RGCs as interference from adjacent cells would be minimum. We have developed an imaging model using a CSLO to study axonal and dendritic degeneration in the Thy1-YFP16Jrs transgenic mice. This technique allows long-term in vivo examination of dendritic and axonal structures in live animal without even the need of local or systemic anesthesia. 
With quantitative analysis of somatic, axonal and dendritic parameters, we characterize the morphology of RGCs, describe the patterns of axonal and dendritic degeneration, identify morphological predictors for cell survival, and estimate the rate of dendritic shrinkage after optic nerve crush.

\section{METHODS}

Animals. Three to 6-month-old Transgenic mice in which Thy-1 promoter sequences drive expression of the enhanced yellow fluorescent protein (YFP), designated B6.Cg-TgN (Thy1-YFP) 16Jrs, were generated by Feng and assoicates. ${ }^{6}$ Breeding pairs of Thy1-YFP16Jrs mice were obtained from the Jackson laboratory (Bar Harbor, ME). The environment was kept at $21^{\circ} \mathrm{C}$ with a 12 -hour light and 12 -hour dark cycle. All mice were fed with ad libitum. Animals used in this study were treated in accordance with the ARVO Statement for the Use of Animals in Ophthalmic and Vision Research.

Optic Nerve Crush. Mice were anesthetized by intraperitoneal injection of ketamine $(100 \mathrm{mg} / \mathrm{kg}$, Ketaset, Fort Dodge Animal Health, Fort Dodge, IA) and xylazine $(9 \mathrm{mg} / \mathrm{kg}$, TranquiVed, Vedco, Inc., St. Joseph, MO). Limbal conjunctival peritomy was performed in the inferior region and gently peeled back to allow access to the posterior region of the globe. The optic nerve was then exposed through a small window made between the surrounding muscle bundles and fatty tissue by gentle blunt dissection. Care was taken not to damage muscles or the vortex veins. At a site approximately $1 \mathrm{~mm}$ posterior to the globe, the optic nerve was clamped using a pair of Dumant No. 5 self-closing tweezers (Ted Pella Inc., Redding, CA) for 2 to 3 seconds. After this procedure, antibiotic ointment was applied to the surgical site. In the postoperative period, mice exhibited normal eating and drinking behavior.

In vivo confocal scanning laser ophthalmoscope (CSLO) imaging. A commercially available CSLO unit (HRA2, Heidelberg Engineering, GmbH, Dossenheim, Germany) was used for RGCs imaging. A 55 degree wide field lens was mounted to the camera to increase the field of view of the fundus. The scan rate of 
the HRA2 was 16 frames per second. Eye-tracking (a retinal recognition technology enabling the same retinal location "locked on") was activated during imaging. Pupils were dilated with topical mydriatic agents (tropicamide and phenylephrine, $0.5 \%$ each) to about $2 \mathrm{~mm}$ in diameter. Fifteen images at the same retinal location were captured, averaged automatically by the built-in software to augment the signal-to-noise ratio and simultaneously displayed on a computer screen. Each retinal frame represents approximately $40 \%$ of total retinal area at an optical resolution $5 \mu \mathrm{m}$ to $10 \mu \mathrm{m}$. A 10 second break was given for every 15 seconds of imaging to allow eye blinking and keeping the corneal surface moist. Photobleaching or phototoxicity was not observed as there was no detectable change in dendritic or axonal morphology even after imaging at the same laser intensity for 30 minutes.

Confocal laser scanning microscopy on retinal whole mount. Mice were anesthetized as described and exsanguinated by perfusion with oxygenated mammalian Ringer's solution containing lidocaine hydrochloride $(0.1 \mathrm{mg} / \mathrm{ml}$, Xylocaine; Astra USA, Inc., Westborough, MA) and heparin sodium (500 units/ml, Heparin; Elkins-Sinn, Inc., Cherry Hill, NJ). Transcardial perfusion was then continued with fixative (approximately $20 \mathrm{ml}$ of $4.0 \%$ paraformaldehyde in $0.1 \mathrm{M}$ phosphate buffer at $\mathrm{pH} 7.4$ ). Retinas were dissected after identifying the inferior area of the retina by the inferior position of the ophthalmic artery. They were placed in fixative for 3-hours, rinsed in $0.1 \mathrm{M}$ phosphate buffer, and then mounted flat on a glass slide. A cover slip was mounted using Fluoromount G. The RGCs were imaged at $20 \mathrm{X}$ magnification by a confocal laser scanning microscope (Nikon C1 Confocoal system, Tokyo, Japan).

Immunohistochemistry. The retina was incubated in a blocking solution containing $10 \%$ normal goat serum (NGS), $2 \%$ bovine serum albumin, and $0.5 \%$ Triton $\mathrm{X}-100$ in phosphate-buffered saline (PBS; $\mathrm{pH}$ 7.4). The mouse anti-SMI-32, a monoclonal antibody against nonphosphorylated neurofilament $H$, was purchased from Sternberger Monoclonals. The primary antibody (1:100) was diluted in 5\% NGS, $1 \%$ BSA, 0.3\% Triton X-100 in PBS and applied for 4 days. Secondary antibody conjugated with rhodamine red 
(1:400, Jackson ImmunoResearch Laboratories) was applied for 2 hours. The retina was mounted in GBmount (Golden Bridge Life Science, Mukilteo, WA).

In vivo Retinal Ganglion Cell Morphometry. The CSLO images were exported to a computer for image analysis with a program written in MATLAB R2007a (The MathWorks, Inc., Natick, MA).computing language environment. The program required manual identification of the dendritic branches and outlining of the cell body margin. Eight parameters were then automatically measured in the RGCs. These include (i) cell body area - the area bounded by the cell body contour, (ii) axon diameter - the thickness of the axon segments nearest to the cell body, (iii) dendritic field - the area bounded by connected line segments joining the ends of all the terminal dendritic branches, (iv) number of terminal branches - total number of terminal dendritic ends, (v) total dendritic branch length - the sum of the lengths of all dendrites, (vi) distance from the optic disc, (vii) branching complexity (modified Sholl Analysis $)^{10}$ - traverses of a series of concentric circles with increasing radii were drawn at the center of the cell body, and (viii) symmetry. Branching complexity was measured as the mean of the function $N(r)$ representing the number of intersections of the skeletonized dendrites $(\mathrm{N})$ for each of the concentric circles with radius $(r)$. The mean of $N(r)$ was defined as dividing the definite integral of $N(r)$ by $\left(r_{\max }-r_{\min }\right)$, where the interval $\left[r_{\min }, r_{\max }\right]$ was chosen in which $N(r)$ was larger than zero within the intervals. Symmetry was the distance between cell body and dendritic arbor centers of mass / dendritic arbor average radius. All measurements were performed by a single observer. Good measurement reliability was found in all 8 parameters with intraclass correlation coefficients ranged between 0.928 and 0.998 . Retinal image calibration was based on a schematic eye model for the C57BL / 6J mouse derived by Remtulla and Hallett. ${ }^{11}$ It was estimated that 1 degree of field is subtended by $30 \mu \mathrm{m}$ of retina at $\lambda=$ $488 \mathrm{~nm}$. 
Cluster Analysis. Cluster analysis was performed with SPSS 16.0 (SPSS Inc. Chicago, April, 2008). Morphological parameters that were not normally distributed were first transformed by taking natural log. Parameters were then standardized using the $z$ scores to minimize the effect of differences in measurement scales. Ward's method was used to define the sum of squared Euclidean distances between RGCs. The agglomeration coefficient was identified in a dendrogram to determine the optimal grouping in the classification (Supplementary Figure).

Statistical Analysis. Statistical analysis was performed with Stata (version 10.0, StataCorp., College Station TX). The survival function of the 6 RGC clusters was evaluated by the Kaplan-Meier estimator and compared with the log-rank test after animal stratification. Baseline morphological parameters were analyzed with the Cox proportional hazards regression model to determine the predictors of RGC survival (total dendritic branch length and dendritic field were analyzed separately because of collinearity). As the survival response of individual RGCs is unlikely to be independent within a mouse, a share frailty model was used to adjust for the effect of within-animal correlation. In the survival analysis, the RGC survival endpoint was defined as complete loss of florescent signal. A linear mixed model was used to estimate the rate of dendritic shrinkage. The model was fitted with fixed coefficients (fixed effects) on baseline dendritic field, age at the time of optic nerve crush, follow-up time, distance away from the optic disc, and the interaction between follow-up time and baseline dendritic field, and distance from the optic disc, with random intercepts and coefficients (random effects) at both the mouse and the eye levels (eye nested within mouse) for the effect of time. $\mathrm{P}<0.05$ is considered to be statistically significant. 


\section{RESULTS}

In vivo Imaging with Confocal Scanning Laser Ophthalmoscope

The $488 \mathrm{~nm}$ pumped semiconductor laser in the CSLO was used to detect the fluorescent signal in the RGCs (488 $\mathrm{nm}$ excitation, $500 \mathrm{~nm}$ detection). The imaging procedure was performed with one technician gently holding the animal and another operating the CSLO (supplementary video). Contact lenses were not required and no topical or systemic anesthesia was applied. A retinal montage constructed from 12 image captures is shown in Figure 1A. Although the microglial and displaced amacrine cells may exhibit dendritic structures similar to the RGCs, the fluorescent cells can be unambiguously identified as the RGCs since the axons are clearly visible emerging from the cell bodies and converging toward the optic disc. It is notable that the dendritic and axonal structures may not be clearly visualized in all RGCs in the CSLO images because the cell body, dendrites and axon of an RGC may not reside on the same retinal plane. The CSLO is limited in imaging multiple focal planes with the same capture. In this study, only fluorescent cells with clear dendritic and axonal structures visualized in vivo were analyzed. The number and the branching of dendritic arborization imaged by the CSLO has direct correspondence with those observed in retinal wholemount using a confocal scanning laser microscope (Figure 1B) although the orientation and spatial arrangement of dendritic trees appeared slightly different. This could be related to changes in tissue architectures during dissection and processing of the retina. The in vivo imaging technique provides real-time morphometry of live RGCs which is not possible with conventional histological analysis.

In vivo Morphometry and Classification of Retinal Ganglion Cells

A total of 125 RGCs from 16 retinas with clear axonal and dendritic structures visualized by the CSLO were analyzed. On average, 11 RGCs were analyzed in each mouse and 8 RGCs were analyzed in each eye. 
Cluster analysis identified 6 groups of RGCs (Figure 1C) with reference to 8 morphological and anatomical parameters (cell body size, axonal diameter, dendritic field size, ending branch number, total dendritic branch length, symmetry, branching complexity (Sholl analysis), and distance from the optic disc) (see Methods and Figure 2). Group 4 RGCs generally had a large dendritic field $\left(148,678 \pm 36,853 \mu \mathrm{m}^{2}\right.$, mean $\pm \mathrm{SD})$ and soma size $\left(3,180 \pm 940 \mu \mathrm{m}^{2}\right)$, long total dendritic branch length $(4,164 \pm 870 \mu \mathrm{m})$, and were located distant from the optic disc $(2,020 \pm 426 \mu \mathrm{m})$ compared with other cell groups. Although there is no consensus on the morphological criteria for RGC classification, most classification systems have been based on measurement of dendritic field and soma size. ${ }^{12-17}$ With a large soma size and dendrites covering a wide area, Group 4 RGCs have close resemblance to alpha-cells originally described by Boycott and Wassle in cats. ${ }^{12}$ For RGCs in groups 5 and 6 , the dendritic field $\left(37,285 \pm 15,562 \mu \mathrm{m}^{2}\right.$ and $36,380 \pm 11,615 \mu \mathrm{m}^{2}$, respectively) and the number of ending dendritic branches $(17.3 \pm 3.9$ and $16.7 \pm$ 3.4) were the smallest, the total dendritic branch length was the shortest $(1,608 \pm 532 \mu \mathrm{m}$ and $1,617 \pm$ $336 \mu \mathrm{m})$, and the dendritic branching was the least complex. Group 6 RGCs had smaller soma size (1,033 $\left.\pm 293 \mu \mathrm{m}^{2}\right)$ and were located closer to the optic disc (731.1 $\left.\pm 364.9 \mu \mathrm{m}\right)$. These morphological characteristics were similar to those reported in gamma-cells. ${ }^{12}$ The dendrites of beta-cells ramify frequently and cover a relatively smaller area. ${ }^{12}$ These features were found in group 1 RGCs in which they had a medium-sized dendritic field $\left(89,988 \pm 26,457 \mu \mathrm{m}^{2}\right)$, yet also had an increased number of ending branches $(28.6 \pm 4.7)$ and high branching complexity. The morphological measurements were similar in Group 2 and 3 RGCs except the axon diameter, dendritic field and cell body size were generally larger for RGCs in group 3. Most RGCs selected in the analysis belonged to group 5 (33.6\%) whereas group 4 RGCs were the least in number (4.0\%) (Figure 1D).

\section{Dendritic and Axonal Changes in Degenerating Retinal Ganglion Cells}

To study the morphological changes in degenerating RGCs, optic nerve crush was performed on 16 eyes 
of 12 mice. The retinas were serially imaged before optic nerve crush, every other day in the first 4 weeks and at least every week thereafter for 3 to 6 months. Most RGCs demonstrated progressive dendritic shrinkage followed by loss of the axons and the cell bodies ( $n=84,68.3 \%$ ) (Figure 3A). Some RGCs had cell bodies detectable for more than 1 to 3 months in the absence of dendritic and axonal structures ( $n=9,7.3 \%$ ) (Figure 3B). A significant proportion of RGCs ( $n=26,21.1 \%$ ) showed only partial loss of dendritic structures without loss of the axons and the cell bodies at 6 months after the injury (Figure 3C). Immunohistochemical staining with anti-SMI-32 indicated that the dendritic shrinkage was not due to redistribution of cytoplasmic YFP (Figure 4).

Progressive axonal changes were only observed in a few RGCs. In 3 RGCs (2.4\%), Wallerian degeneration with fragmentation and beading of the axon was observed following progressive dendritic shrinkage (Figure $5 \mathrm{~A})$. In one RGC $(0.8 \%)$, the proximal axon showed retraction and bulb formation before cell death (Figure 5B).

\section{Morphological Predictors of Retinal Ganglion Cell Survival after Optic Nerve Injury}

Survival analysis showed that group 4 RGCs had the best survival function and group 6 had the worst (Figure 6A) (the RGC survival endpoint was defined as complete loss of fluorescent signal). There was a significant difference in the survival function between RGCs in group 4 and RGCs in groups 1, 2, 5 and 6 (log-rank test stratified for mouse, $p=0.038$ ). Among the 8 morphological parameters measured at baseline, the dendritic field, total dendritic branch length and distance from the optic disc were independent predictors of RGC survival in the Cox regression analysis (all with $p \leq 0.035$ ) (Table 1). Improved survival function was generally found in cells with a larger dendritic field, longer total dendritic branch length, and at a longer distance from the optic disc. The probability of survival is increased by $13.1 \%, 4.4 \%$ and $6.0 \%$ for every $10,000 \mu \mathrm{m}^{2}$ increase in dendritic field, every $100 \mu \mathrm{m}$ increase in total dendritic branch length, and every $100 \mu \mathrm{m}$ increase in distance away from the optic disc, respectively. 
Baseline cell body size, axon diameter, symmetry, dendritic branching complexity and the number of ending dendritic branches had no association with RGC survival. It is possible to predict the survival probability of individual RGC with reference to baseline morphological measurements. Figure $6 \mathrm{~B}$ compared the predicted survival probability of RGCs with a large $\left(150,000 \mu \mathrm{m}^{2}\right)$ and a small $\left(35,000 \mu \mathrm{m}^{2}\right)$ dendritic field after standardizing other morphological measurements. At one month after optic nerve crush, the survival probabilities were 0.85 and 0.45 , respectively.

\section{Measuring the Rate of Dendritic Shrinkage}

A linear mixed model was applied to estimate the rate of change of dendritic field of individual RGCs after optic nerve crush. A total of 673 serial dendritic field measurements from 122 RGCs were analyzed. Interaction terms between the time after the crush and the baseline dendritic field, and the distance from the optic disc were included in the model to evaluate their influences on the rate of dendritic shrinkage. In agreement with the Cox proportional hazards regression analysis, the positive coefficient of the interaction terms $\left(1.34 \times 10^{-7}\right.$ and $7.60 \times 10^{-6}$, respectively) (Table 2$)$ indicates that RGCs with a larger baseline dendritic field or at a longer distance from the optic disc had a slower rate of dendritic shrinkage. For example, the rate of dendritic shrinkage of a RGC with a dendritic field $10,000 \mu \mathrm{m}^{2}$ and close to the optic nerve head $(600 \mu \mathrm{m})$ would be $-3.51 \%$ [-0.041 (coefficient of time in day) $\times 1+$ $\left.\left.1.34 \times 10^{-7} \times 10,000+7.60 \times 10^{-6} \times 600\right)\right]$ per day. This is in contrast to a RGC with a larger dendritic field $\left(150,000 \mu \mathrm{m}^{2}\right)$ at the same location in which the estimated rate of dendritic shrinkage is $-1.63 \%$ per day. The rate of dendritic shrinkage in relation to baseline dendritic field for RGCs at $2000 \mu \mathrm{m}$ and $600 \mu \mathrm{m}$ away from the optic nerve head was modeled in Figure $6 C$ and $6 D$, respectively. RGCs with large dendritic field distant from the optic disc have the slowest rate of dendritic shrinkage after optic nerve crush. 


\section{DISCUSSION}

Being able to serially monitor cellular morphology has been a major challenge in studying RGC degeneration. Animal models designed for in vivo imaging almost always required systemic anesthesia, which is undesirable for frequent, long term assessment. In addition, clouding of the lens associated with systemic anesthesia could impair visualization of the fundus. ${ }^{18}$ Walsh and Quigley demonstrated the feasibility of in vivo imaging of dendritic processes of RGCS in anaesthetized Thy1-YFP16Jrs transgenic mice with a modified confocal laser scanning microscope. ${ }^{19}$ However, dendritic arborization visualized in this model was not as distinct as fixed tissue imaging and this limited the potential for quantitative morphological analysis. Gray et al showed that it is possible to image the dendritic arborization in macaques using an adaptive optics scanning laser ophthalmoscope with retrograde labeling. ${ }^{20}$ However, the labeling with rhodamine is transitory. Long term in vivo analysis of dendritic damage in neuronal degeneration has not been demonstrated previously.

The key advantage of this real-time CSLO imaging is that it provides an efficient and non-invasive approach for capturing serial retinal images at the same location over months. In particular, there is consistently high image quality that allows detailed morphological analysis and classification of RGCs, monitoring of axonal and dendritic changes, evaluation of survival probability, and measurement of the rate of dendritic shrinkage. Imaging is performed with gentle holding of the animal without using any local or systemic anesthetic agent, which may compromise animal survival particularly when serial capture is warranted. The feasibility of long term monitoring of dendritic and axonal changes for more than 6 months has been demonstrated in this study (Figure 3C). Of note, the YFP expressing RGCs likely represent a random sample of the retina as it has been shown that the expression has no predilection for specific subtypes of RGCs. ${ }^{16}$ 
The confocal imaging technique, however, is limited in evaluating the dendritic stratification in cross-section. It is not yet possible to identify the dendritic spines with the current instrumentation. This information was not available for the cluster analysis thereby limiting finer classification. Nevertheless, it is evident from the Kaplan Meier analysis that the current morphological classification is related to RGC survival (Figure 6A). In fact, we are able to predict the survival probability and estimate the rate of dendritic shrinkage after optic nerve crush based on the size of dendritic field and the distance from the optic disc measured at baseline. To our knowledge, this is the first study measuring and modeling progressive dendritic shrinkage in vivo. We showed that RGC degeneration began with the dendritic trees, followed by loss of the axons and finally the cell bodies (Figure 3). The rate and the degree of dendritic shrinkage, however, were variable among individual neurons. RGCs with larger dendritic field showed a higher survival probability and a slower rate of dendritic shrinkage. Notably, although the procedure of optic nerve crush had been standardized by performing the crush at a site approximately $1 \mathrm{~mm}$ posterior to the globe and clamping for 2-3 seconds, it is possible that the variable rate and degree of dendritic shrinkage could also be related to minute variation in the position, duration, and clamping pressure during the procedure.

Progressive axonal changes were only observed in a small proportion of RGCs, suggesting that axonal degeneration is a relatively rapid process. Although mice were not imaged immediately after optic nerve crush, no detectable changes were evident at day 1 after optic nerve crush compared to the baseline. It has been proposed that focal axonal lesions induce Wallerian degeneration of distal axons whereas the proximal axons "dies back". ${ }^{21}$ Using the Thy-1 GFP-S transgenic mice, Kerschensteiner et al showed that spinal cord axons underwent acute axonal degeneration with rapid retraction of proximal axon stumps within 30 minutes after transection followed by Wallerian degeneration of distal axons after 30 hours. $^{1}$ In this study, we found that both Wallerian degeneration (Figure 5A) and axonal retraction (Figure 5B) could take place in proximal axons. And this could occur even at a month after optic nerve 
crush. While the mechanism triggering the axonal degeneration remains to be elucidated, a common molecular pathway involving Wallerian degeneration slow (WLD-S) has been suggested for Wallerian degeneration and axonal retraction. ${ }^{1,22}$ The delayed axonal degeneration in the RGCs implies a potential therapeutic window after optic nerve injury. In some RGCs, the cell bodies survived despite the absence of detectable axons and dendritic arborization (Figure 3b). Restoring axonal and dendritic structures would be a key area for research on neuronal regeneration.

It has been demonstrated that axon degeneration precedes cell death in neurodegenerative diseases. ${ }^{23,24}$ We found that dendritic shrinkage could be evident even before axon degeneration and could thus serve as an early sign for neuronal damage. Being able to visualize the changes in dendritic arborization and measure the rate of dendritic shrinkage provides a new paradigm to study the mechanism of neurodegeneration and may provide new insights in testing novel therapies for neuroprotection.

\section{REFERENCES}

1. Kerschensteiner M, Schwab ME, Lichtman JW, et al. In vivo imaging of axonal degeneration and regeneration in the injured spinal cord. Nat Med. 2005;11: 572-7.

2. Misgeld T, Nikic I, Kerschensteiner M. In vivo imaging of single axons in the mouse spinal cord. Nat Protoc. 2005;2:263-8.

3. Sabel BA, Engelmann R, Humphrey MF. In vivo confocal neuroimaging (ICON) of CNS neurons. Nat Med. 1997;3:244-7.

4. Thanos S, Indorf L, Naskar R. In vivo FM: using conventional fluorescence microscopy to monitor retinal neuronal death in vivo. Trends Neurosci. 2002;25:441-4. 
5. Köbbert C, Apps R, Bechmann I, et al. Current concepts in neuroanatomical tracing. Prog Neurobiol. 2000;62:327-51.

6. Feng $\mathrm{G}$, Mellor $\mathrm{RH}$, Bernstein $\mathrm{M}$, et al. Imaging neuronal subsets in transgenic mice expressing multiple spectral variants of GFP. Neuron. 2000;28:41-51.

7. Leung CK, Lindsey JD, Crowston JG, et al. In vivo imaging of murine retinal ganglion cells. J Neurosci Methods. 2008;168:475-8.

8. Leung CK, Lindsey JD, Crowston JG, et al. Longitudinal Profile of Retinal Ganglion Cell Degeneration After Optic Nerve Crush - A Blue-Light Confocal Scanning Laser Ophthalmoscope Imaging Study. Invest Ophthalmol Vis Sci. 2008;49:4898-902.

9. Leung CK, Lindsey JD, Chen L, et al. Longitudinal profile of retinal ganglion cell damage assessed with blue-light confocal scanning laser ophthalmoscopy after ischemic reperfusion injury. $\mathrm{Br} \mathrm{J}$ Ophthalmol. 2009;93:964-8.

10. Ristanovic D, Milosević NT, Stulić V. Application of modified Sholl analysis to neuronal dendritic arborization of the cat spinal cord. J Neurosci Methods. 2006;158:212-8.

11. Remtulla S, Hallett PE. A schematic eye for the mouse, and comparisons with the rat. Vision Res. $1985 ; 25: 21-31$.

12. Boycott BB, Wässle $\mathrm{H}$. The morphological types of ganglion cells of the domestic cat's retina. J Physiol. 1974;240:397-419.

13. Stone J, Clarke R. Correlation between soma size and dendritic morphology in cat retinal ganglion cells. J Comp Neurol. 1980;192:211-7.

14. Perry VH. The ganglion cell layer of the retina of the rat: a Golgi study. Proc R Soc Lond B Biol Sci. 1979;204:363-75.

15. Doi M, Uji Y, Yamamura H. Morphological classification of retinal ganglion cells in mice. J Comp Neurol. 1995;356:368-86. 
16. Coombs J, van der List D, Wang GY, et al. Morphological properties of mouse retinal ganglion cells. Neuroscience. 2006;140:123-36.

17. Sun W, Li N, He S. Large-scale morphological survey of mouse retinal ganglion cells. J Comp Neurol. 2002;451:115-26.

18. Calderone L, Grimes P, Shalev M. Acute reversible cataract induced by xylazine and by ketaminexylazine anesthesia in rats and mice. Exp Eye Res. 1986;42:331-7.

19. Walsh MK, Quigley HA. In vivo time-lapse fluorescence imaging of individual retinal ganglion cells in mice. J Neurosci Methods. 2008;169:214-21.

20. Gray DC, Wolfe R, Gee BP, et al. In vivo imaging of the fine structure of rhodamine-labeled macaque retinal ganglion cells. Invest Ophthalmol Vis Sci. 2008;49:467-73.

21. Coleman M. Axon degeneration mechanisms: commonality amid diversity. Nat Rev Neurosci. $2005 ; 6,889-98$

22. Lunn ER, Perry VH, Brown MC, et al. Absence of Wallerian Degeneration does not Hinder Regeneration in Peripheral Nerve. Eur J Neurosci. 1989; 1:27-33.

23. Stokin GB, Lillo C, Falzone TL, et al. Axonopathy and transport deficits early in the pathogenesis of Alzheimer's disease. Science. 2005;307:1282-1288.

24. Li H, Li SH, Yu ZX, et al. Huntingtin aggregate-associated axonal degeneration is an early pathological event in Huntington's disease mice. J. Neurosci. 2001;21:8473-8481. 


\section{FIGURE LEGENDS}

Figure 1. In vivo imaging with a confocal scanning laser ophthalmoscope (HRA2, Heidelberg Engineering, $\mathrm{GmbH}$, Dossenheim, Germany) showing the axonal and dendritic structures of the retinal ganglion cells (RGCs) in transgenic Thy-1 YFP mice. (A) A retinal montage was constructed from 12 image captures in different retinal areas of a healthy transgenic Thy-1 YFP mouse. The fluorescent cells can be distinctively identified as RGCs since the axons are clearly visible emerging from the cell bodies and running towards the optic disc. It is notable the dendritic and axonal structures may not be clearly visualized in all RGCs in the CSLO images because the cell body, dendrites and axon of an RGC may not reside on the same retinal plane. (B) There is a direct correspondence in the branching number and pattern of dendritic arborization between live image capture (left) and image obtained with a confocal laser scanning microscope on a retinal whole mount (right). (C) Cluster analysis on 125 RGCs with clear axonal and dendritic structures visualized by the CSLO identified 6 morphological groups with reference to (1) cell body size, (2) axonal diameter, (3) dendritic field, (4) ending branch number, (5) total dendritic branch length, (6) symmetry, (7) branching complexity (Sholl analysis), and (8) distance from the optic disc (See

Figure 2). (D) Among the 6 morphological groups, group 4 RGCs constituted the smallest proportion in the retina whereas one-third of the RGCs belonged to group 5. 
Figure 2. Measurement of 8 morphological and anatomical parameters including (A) cell body size, (B) dendritic field, (C) axonal diameter, (D) ending branch number, (E) total dendritic branch length, (F) symmetry, (G) branching complexity (Sholl analysis), and (H) distance from the optic disc in 6 groups of retinal ganglion cells (RGCs) classified in the cluster analysis. Group 4 RGCs have the largest dendritic field and cell body area, the longest total dendritic branch length, and furthest away from the optic disc. RGCs in group 5 and 6 have the smallest dendritic field and the shortest total dendritic branch length. Group 6 RGCs have the smallest cell body size and they are close to the optic disc. The dendritic branching is most complex in group 1 RGCs and they have the highest number of ending branch. Yet, the dendritic field is medium in size. Group 2 and 3 RGCs are similar in many aspects although the axon diameter, dendritic field and cell body size are generally greater for RGCs in group 3. Error bar standard error of the mean. 
Figure 3. Long term serial in vivo imaging of retinal ganglion cell degeneration after optic nerve crush. (A) Progressive dendritic shrinkage precedes loss of the the axon and the cell body. Loss of dendritic branches and reduction in dendritic field were evident 7 days after optic nerve crush. There was progressive dendritic shrinkage in the following week. The axon became indistinct on day 13 with complete loss of dendrites and cell body on day 15. (B) In another RGC (arrowhead), progressive dendritic shrinkage was observed from day 0 to day 25 with loss of axon on day 17 (arrow). The cell body, however, persisted at the time of last image capture on day 133. The axon remaining on day 133 belonged to another RGC distant from the optic disc. (C) Despite progressive dendritic shrinkage and loss of dendritic branches, this RGC retained some dendritic features. The axon and the cell body were all preserved for more than 6 months. 
Figure 4. Co-localization between yellow fluorescent protein (YFP) expression and anti-SMI-32 immunohistochemical staining in a degenerating retinal ganglion cell imaged by a confocal scanning laser microscope. The retina from a 6-month-old Thy-1 YFP transgenic mouse 12 months after optic nerve crush was dissected and immunolabeled with anti-SMI-32, a monoclonal antibody against nonphosphorylated neurofilament $\mathrm{H}$. The secondary antibodies were conjugated with rhodamine red and the dendritic structures were visualized in red (left panel). The expression of YFP in the retinal ganglion cell (green) (middle panel) co-localized with anti-SMI-32 (right panel) suggesting that dendritic changes visualized through detection of florescent signal was not due to redistribution of cytoplasmic YFP. 
Figure 5. Progressive axonal changes in retinal ganglion cell degeneration. (A) Fragmentation and beading of axon with features compatible with Wallerian degeneration were detected in an RGC on day 39 and lasted for approximately 4 days (day 39 - day 42) after the crush. (B) In another RGC, axonal retraction and bulb formation was found on day 52. On day 57, there was complete loss of dendrites, axon and cell body. 
Figure 6. Estimation of survival probability and rate of dendritic shrinkage of retinal ganglion cells after optic nerve crush. (A) Survival function of RGCs was analyzed by the Kaplan Meier estimator. Group 4 RGCs had a higher survival probability than RGCs in group 2, 5 and 6 (log-rank test stratified for mouse, $\mathrm{p}=0.038$ ). (B) Using a Cox regression analysis model with adjustment of cell body size, axonal diameter, ending branch number, symmetry, branching complexity (Sholl analysis), and distance from the optic disc, it is estimated that RGCs with a dendritic field of $35,000 \mu \mathrm{m}^{2}$ would have exponential decrease in survival probability after optic nerve crush. At one month after the crush, the estimated survival probability is 0.45 . This is in contrast to RGCs with a larger dendritic field $\left(150,000 \mu \mathrm{m}^{2}\right)$ in which the survival probability is improved to 0.85 . The survival endpoint of RGCs was defined as complete loss of fluorescent signal. The rate of dendritic field shrinkage after optic nerve crush was analyzed with a linear mixed model and plotted three-dimensionally with respect to different baseline dendritic field and at $2000 \mu \mathrm{m}$ (C) and $600 \mu \mathrm{m}$ (D) from the optic disc. The baseline dendritic field was set in a range between 30,000 and $160,000 \mu \mathrm{m}^{2}$. The rate of dendritic shrinkage can be estimated from the $z-x$ axes. In general, RGCs with a small dendritic field and located near to the optic disc have an increased rate of dendritic shrinkage. 
Supplementary Figure. Cluster analysis of retinal ganglion cells. The differences between individual retinal ganglion cells (RGCs) were signified by the squared Euclidean distances in the multidimensional space defined by the 8 morphological parameters. RGCs with shorter Euclidean distances were merged together to form the initial groups. The distances between the groups were defined by the sum of the squared distances of individual RGCs within the group (Ward's Method). Different groups were then merged together until there was only one group left and a dendrogram was formed. In the dendrogram, groups merged by shorter distances share more similarities and the agglomeration coefficient described the largest distance among all the groups. A shape rise in the agglomeration coefficient indicates a sudden increase in dissimilarities in the corresponding merging process. The turning point (arrow) in the dendrogram was identified as the optimal number of clusters for preserving the similarities of RGCs within groups. 
Supplementary Video. In vivo imaging was performed with a technician gently holding the mouse without application of topical or systemic anesthetic agent. Another technician adjusted the focus of the confocal scanning laser ophthalmoscope on the retina. Eye-tracking (a retinal recognition technology enabling the same retinal location "locked on") was activated during in vivo imaging to allow simultaneous image averaging. A retinal image with clear visualization of dendritic and axonal structures was captured in less than 5 seconds. 
Table 1 Predictors of retinal ganglion cell survival probability analyzed by a Cox proportional hazard regression model with share frailty.

\begin{tabular}{l|ccccc} 
Parameter & Hazard Ratio & Standard Error & $p$ & $95 \%$ Confidence Interval \\
& & & & Lower & Upper \\
\hline Axon Diameter $(\mu \mathrm{m})$ & 0.995 & 0.040 & 0.906 & 0.922 & 1.074 \\
Symmetry & 2.675 & 2.144 & 0.220 & 0.556 & 12.879 \\
Distance from Optic Disc $($ per $100 \mu \mathrm{m})$ & 0.940 & 0.021 & 0.006 & 0.899 & 0.982 \\
Cell Body $\left(\mu \mathrm{m}^{2}\right.$ ) & 1.000 & 0.0002 & 0.884 & 1.000 & 1.000 \\
*Dendritic Field (per 10,000 $\left.\mu \mathrm{m}^{2}\right)$ & 0.869 & 0.046 & 0.009 & 0.783 & 0.965 \\
*Total Branch Length $($ per $100 \mu \mathrm{m})$ & 0.956 & 0.020 & 0.035 & 0.917 & 0.997 \\
Ending Branch Number & 0.990 & 0.036 & 0.792 & 0.922 & 1.064 \\
Sholl Analysis & 1.033 & 0.093 & 0.718 & 0.866 & 1.233
\end{tabular}

*Total dendritic branch length and dendritic field were analyzed separately because of collinearity. 
Table 2 Estimation of rate of dendritic shrinkage using a linear mixed model with fixed coefficients (fixed effects) including baseline dendritic field, age at the time of optic nerve crush, follow-up time, distance away from the optic disc, and the interaction between baseline dendritic field / distance away from the optic disc and follow-up time, with random intercepts and coefficients (random effects) at both the mouse and the eye levels (eye nested within mouse) for the effect of time.

\begin{tabular}{l|ccccc} 
& Coefficient & Standard Error & $P$ & \multicolumn{2}{c}{$95 \%$ Confidence Interval } \\
& & & & Lower & Upper \\
\hline Age (day) & 0.002 & 0.001 & 0.114 & -0.0006 & 0.005 \\
Time (day) & -0.041 & 0.006 & $<0.001$ & -0.052 & -0.030 \\
Baseline Dendritic Field $\left(\mu \mathrm{m}^{2}\right)$ & $7.34 \times 10^{-7}$ & $5.22 \times 10^{-7}$ & 0.160 & $-2.90 \times 10^{-7}$ & $1.76 \times 10^{-6}$ \\
Distance from Optic Disc $(\mu \mathrm{m})$ & $5.08 \times 10^{-5}$ & $3.34 \times 10^{-5}$ & 0.128 & $-1.47 \times 10^{-5}$ & $1.16 \times 10^{-4}$ \\
Baseline Dendritic Field X Time & $1.34 \times 10^{-7}$ & $5.20 \times 10^{-8}$ & 0.010 & $3.22 \times 10^{-8}$ & $2.36 \times 10^{-7}$ \\
Distance from Optic Disc X Time & $7.60 \times 10^{-6}$ & $3.29 \times 10^{-6}$ & 0.021 & $1.15 \times 10^{-6}$ & $1.40 \times 10^{-5}$ \\
Constant & 0.465 & 0.172 & 0.007 & 0.127 & 0.802
\end{tabular}


Figure 1

(A)

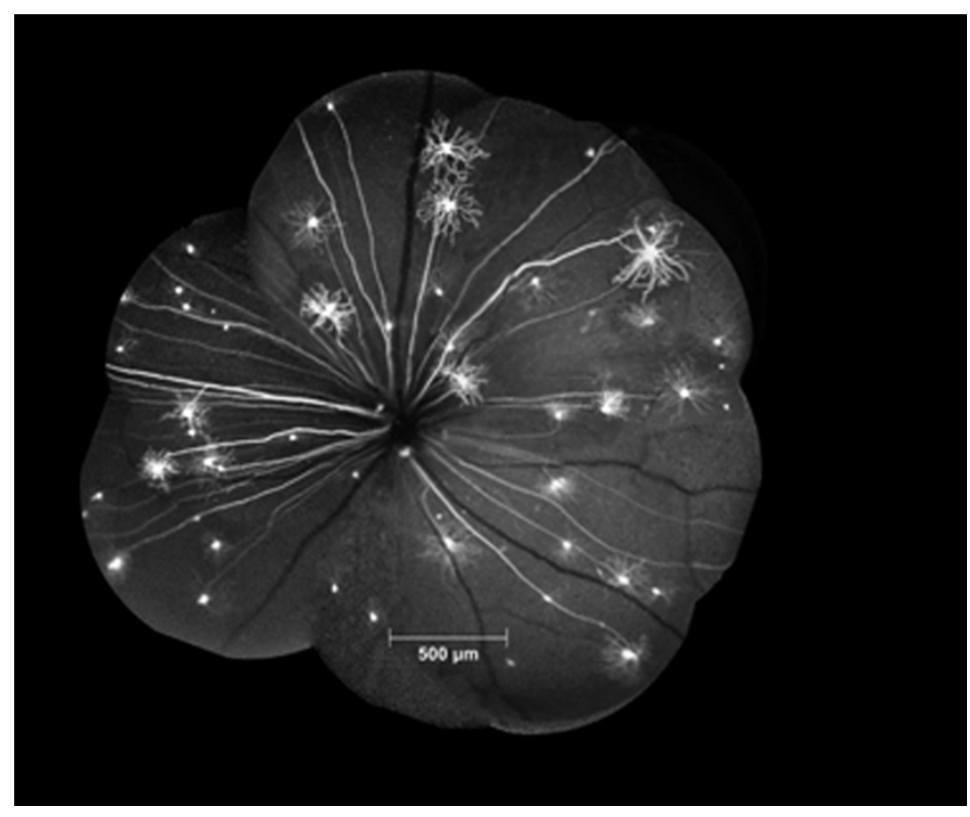

(B)
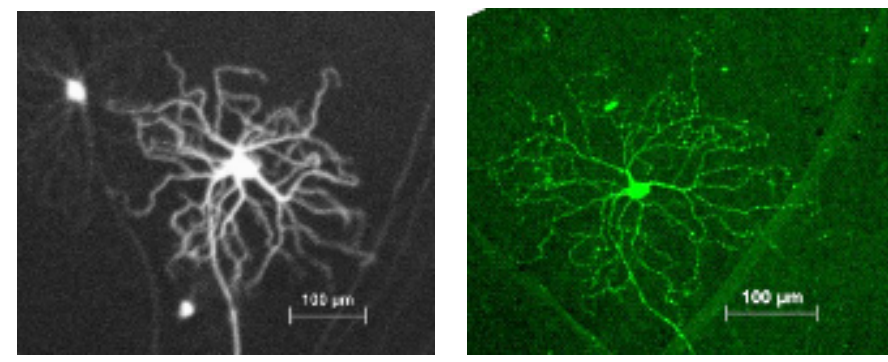

(C)

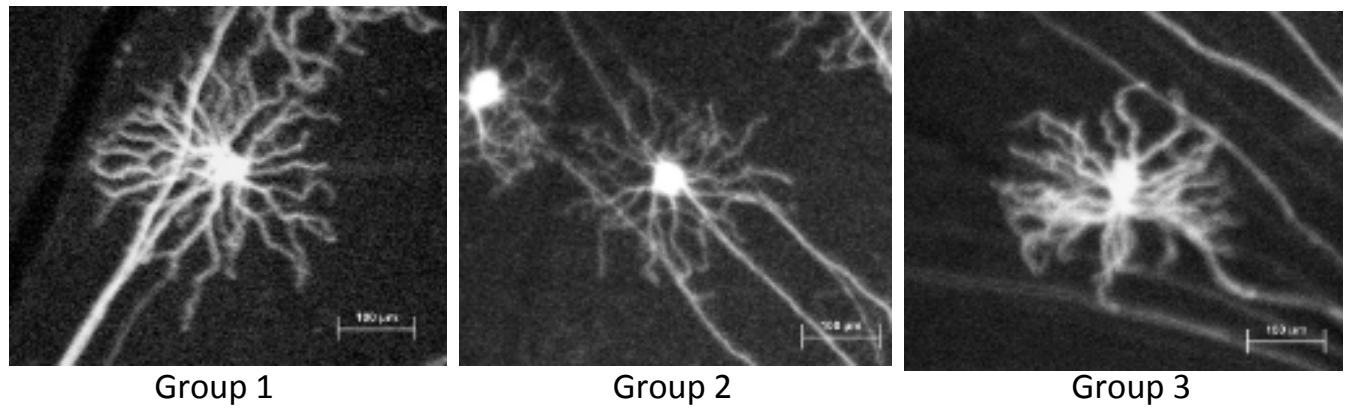

Group 1

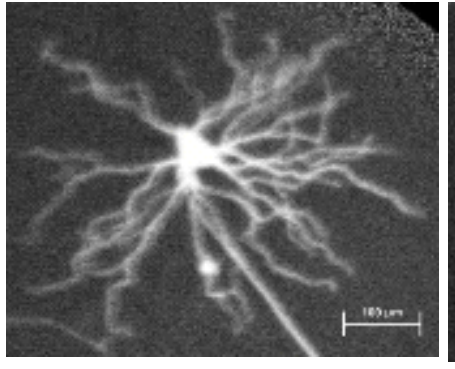

Group 4

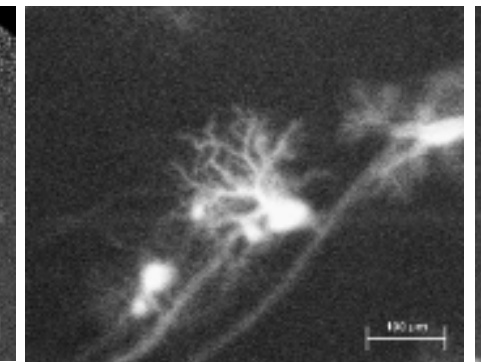

Group 5

(D)

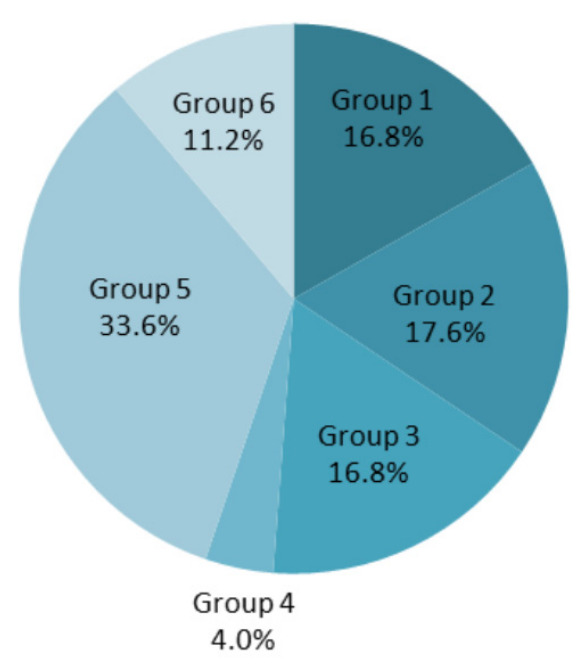


Figure 1

(c)

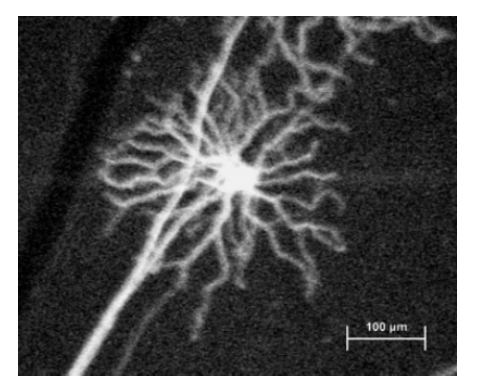

Group 1

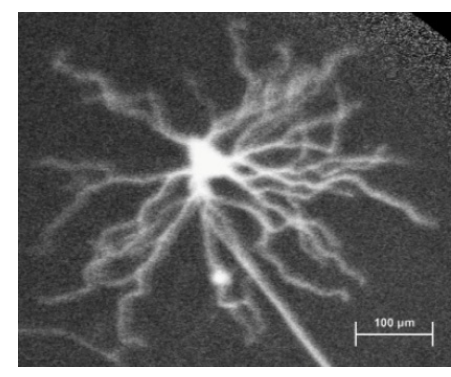

Group 4

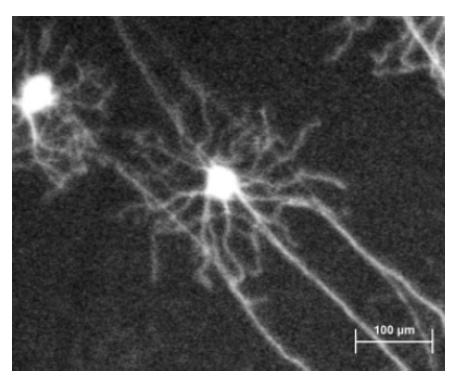

Group 2

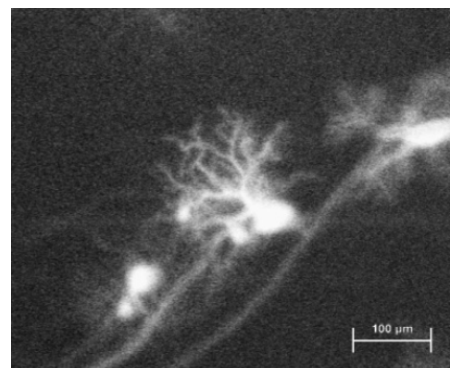

Group 5

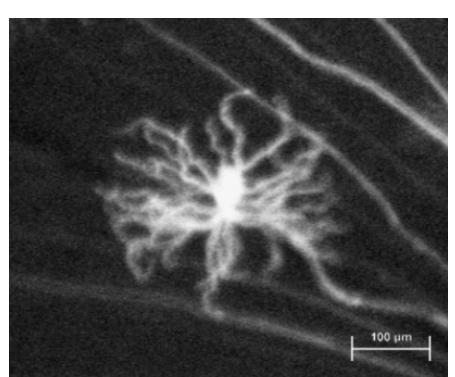

Group 3

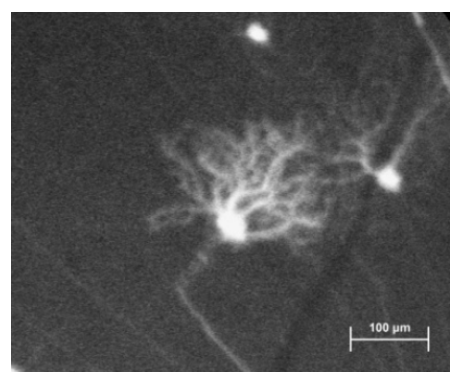

Group 6

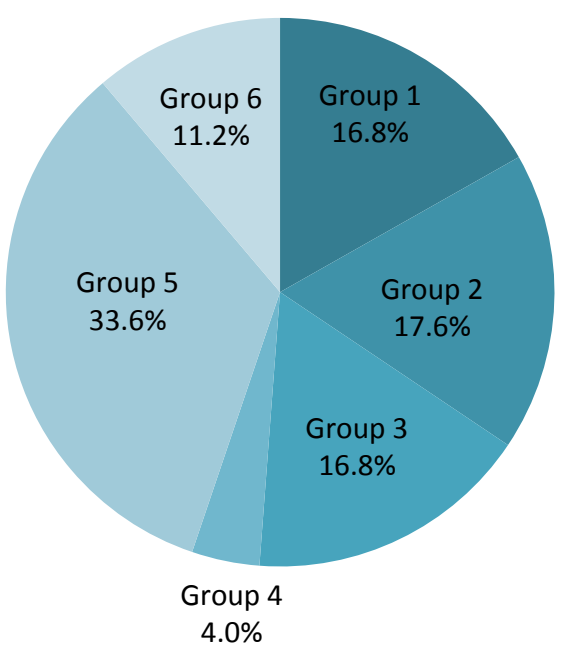


Figure 2
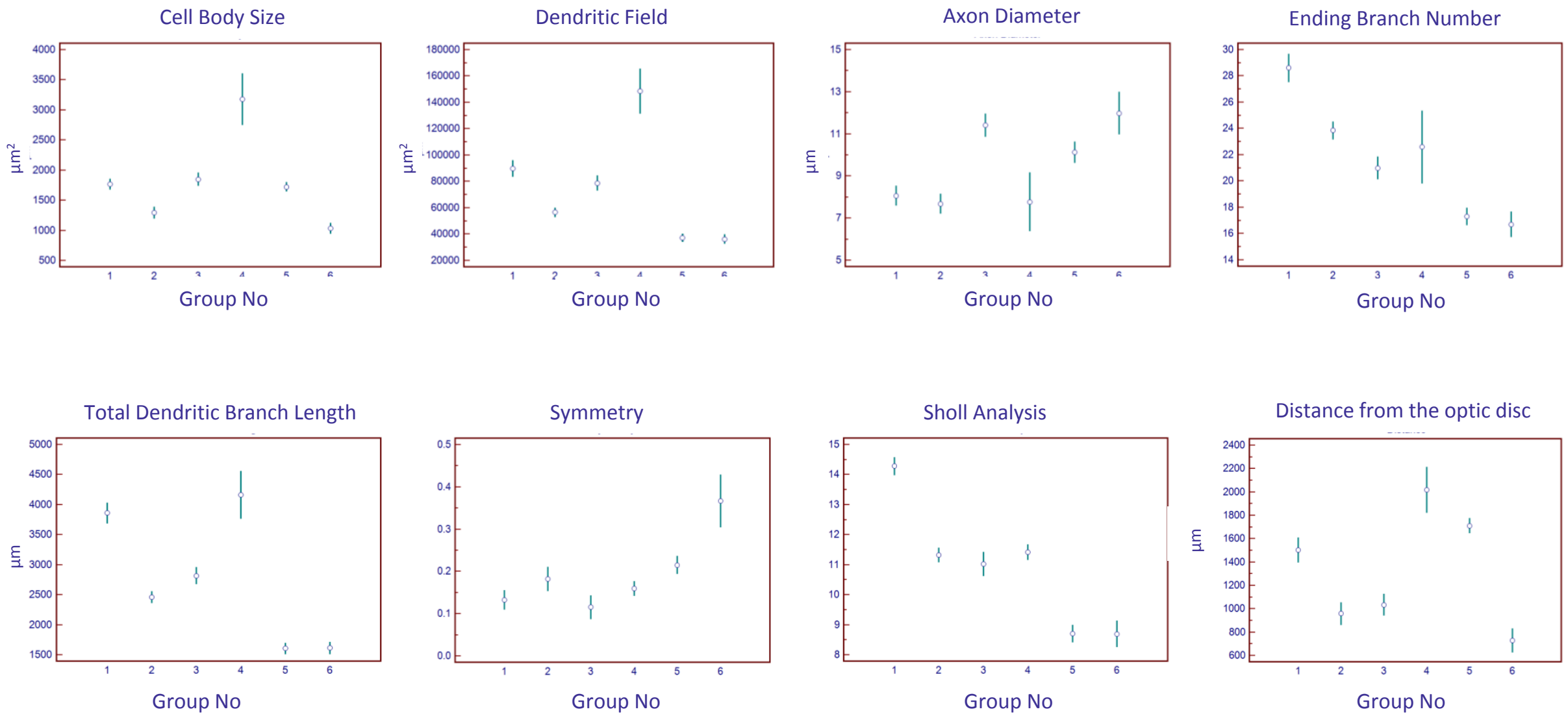
Figure 3

(a)

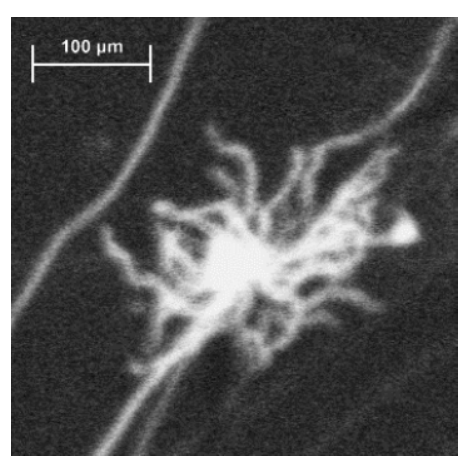

Baseline

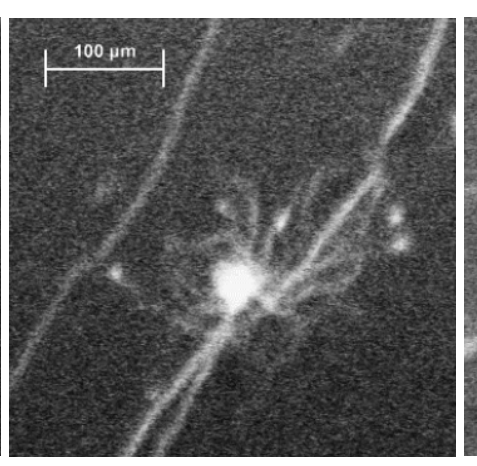

Day 7

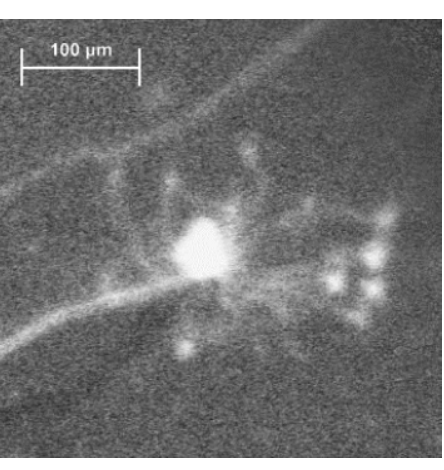

Day 11

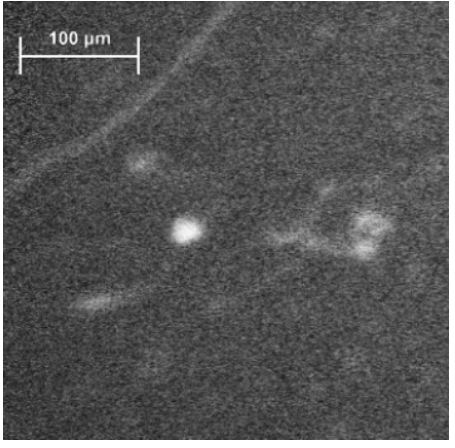

Day 13

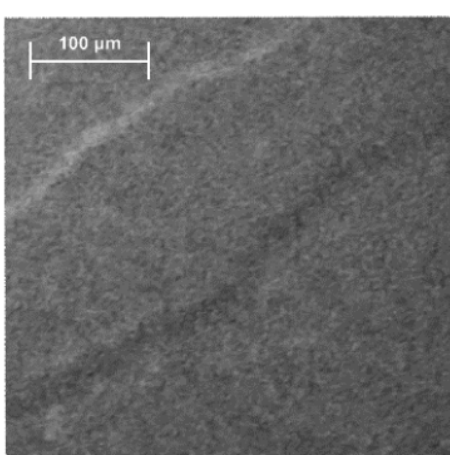

Day 15 
(b)

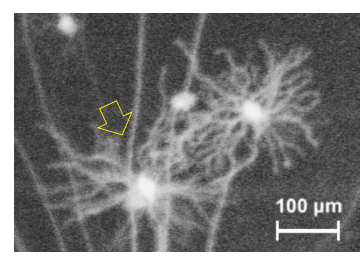

Baseline

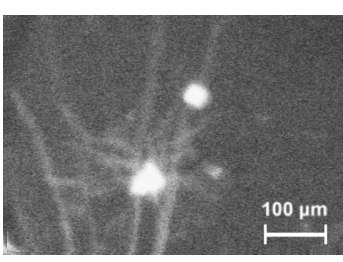

Day 8

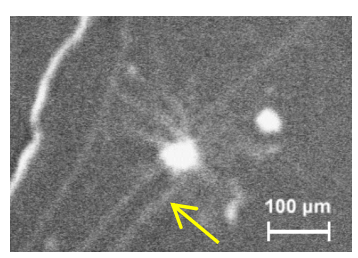

Day 15

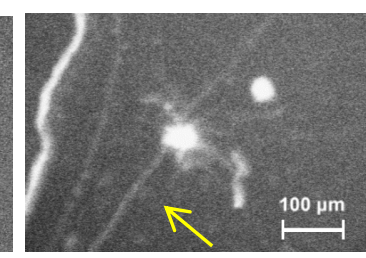

Day 17

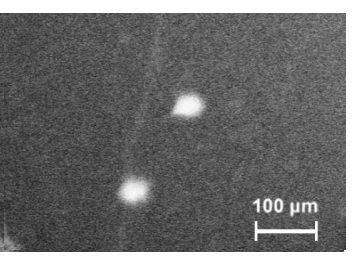

Day 25

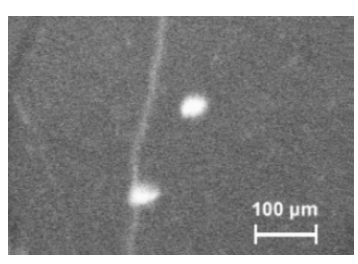

Day 133

(c)

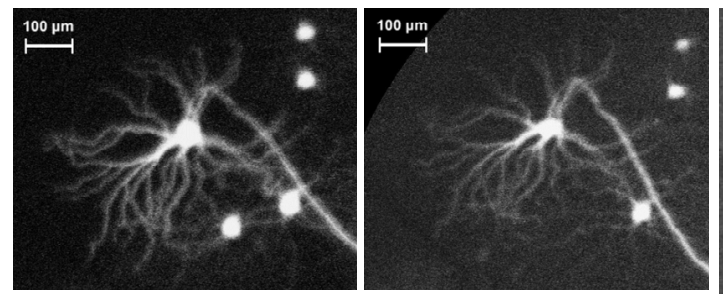

Baseline

Day 7

(d)

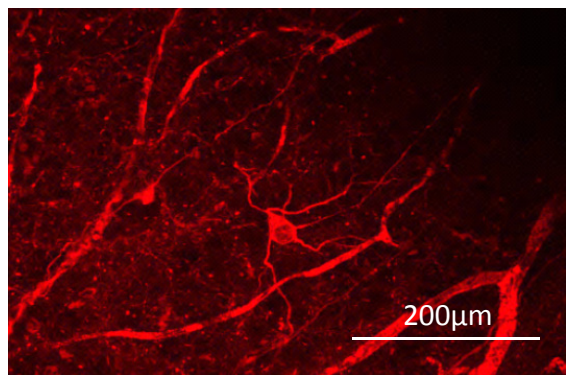

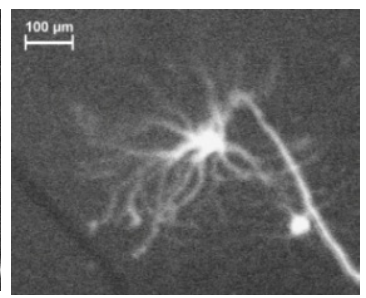

Day 15

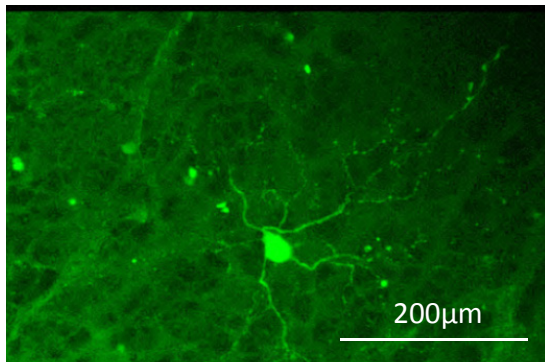

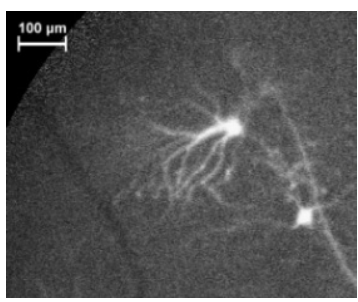

Day 50

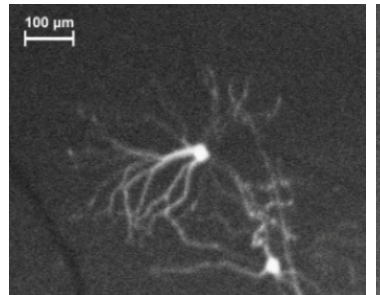

Day 94

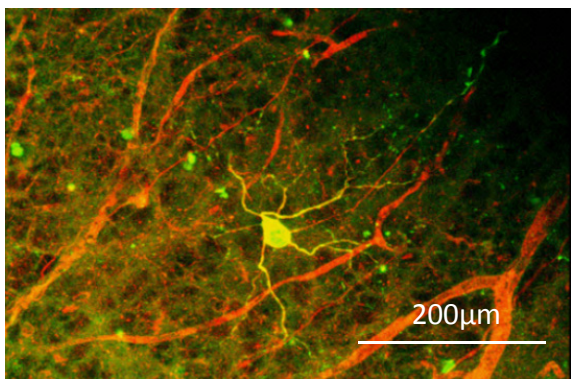

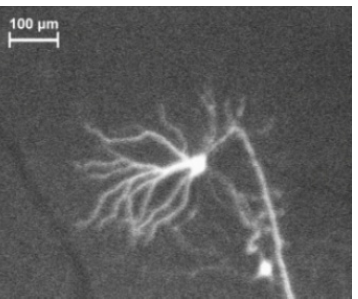

Day 185 
Figure 4

(a)

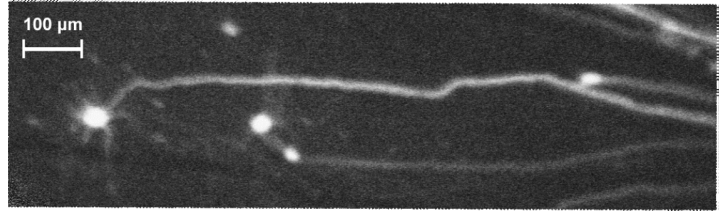

Day 18

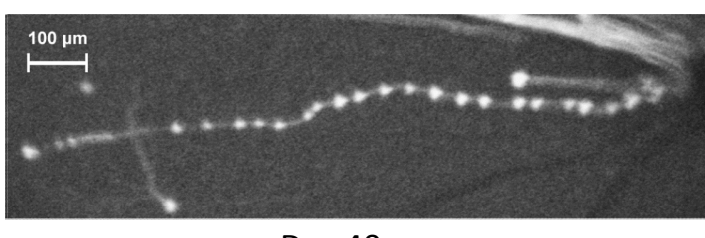

Day 40

(b)

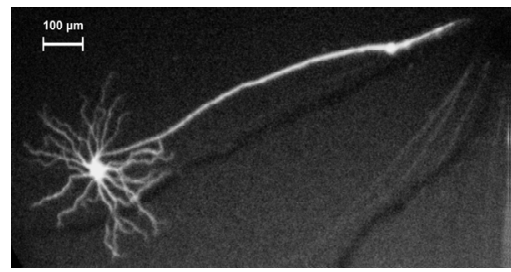

Baseline

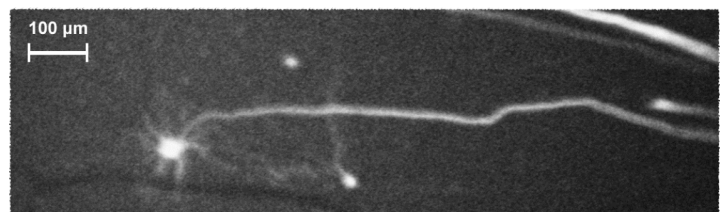

Day 33

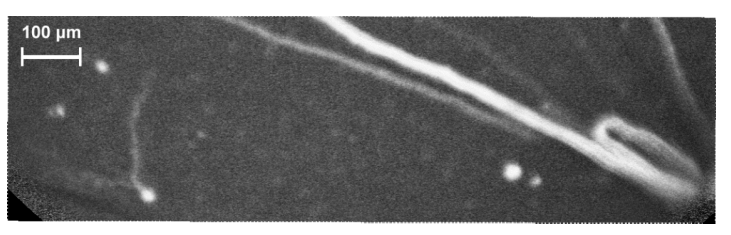

Day 42

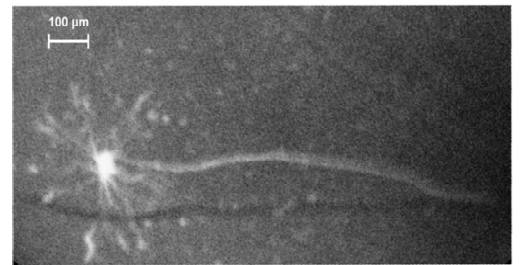

Day 17

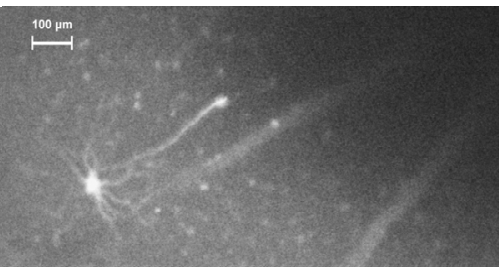

Day 52

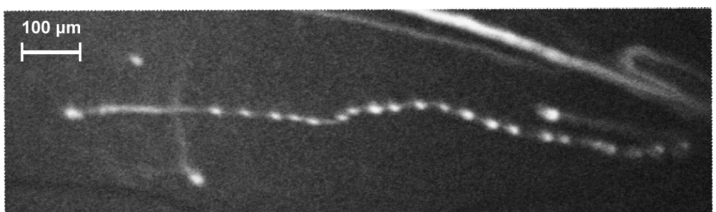

Day 39

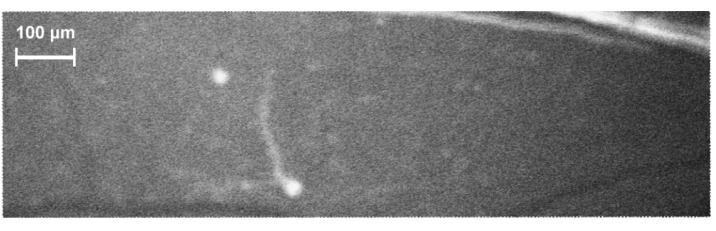

Day 47

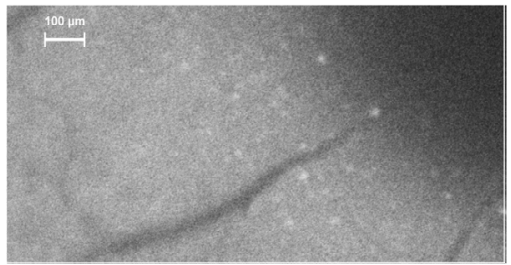

Day 57 
Figure 5

(a)

(b)

Retinal ganglion cell group
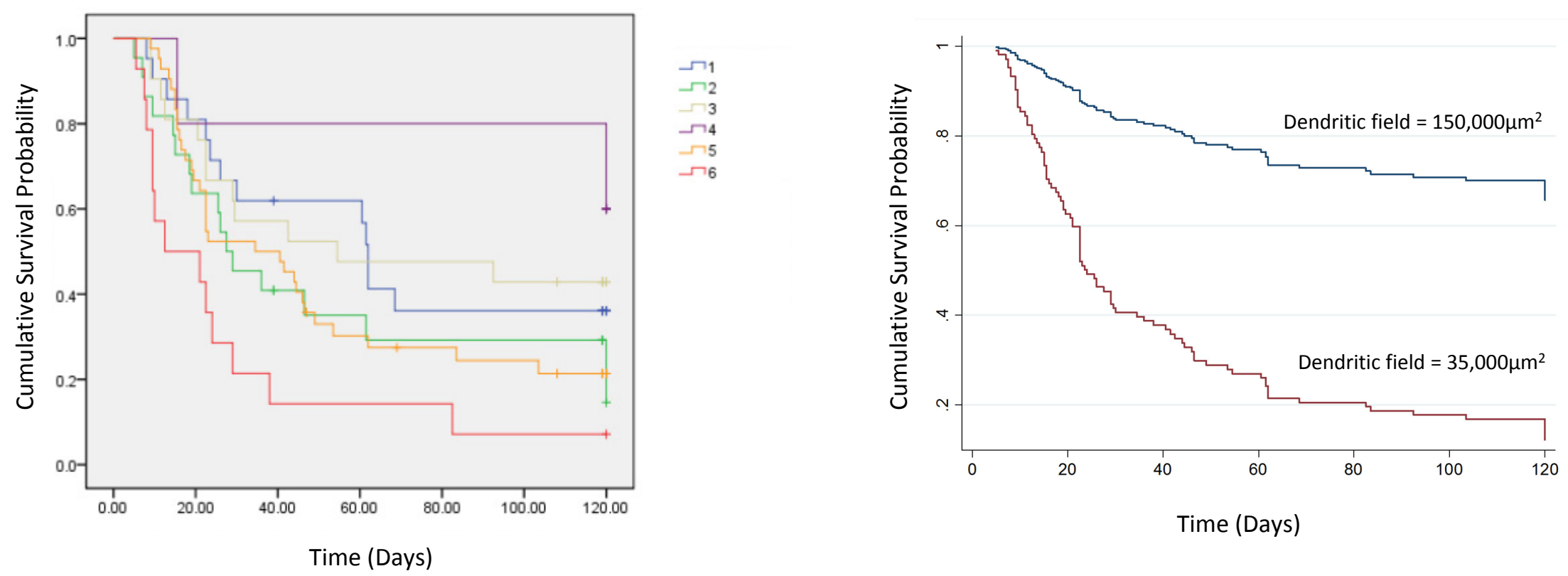
Figure 6

(a)

(b)
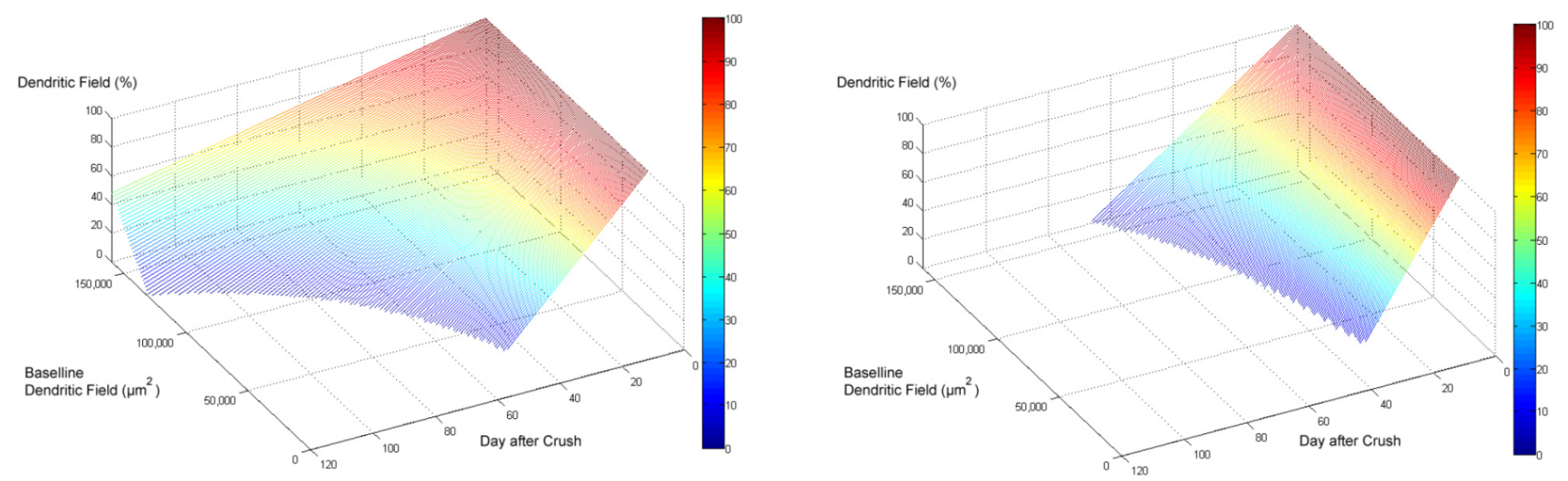
Supplementary Figure

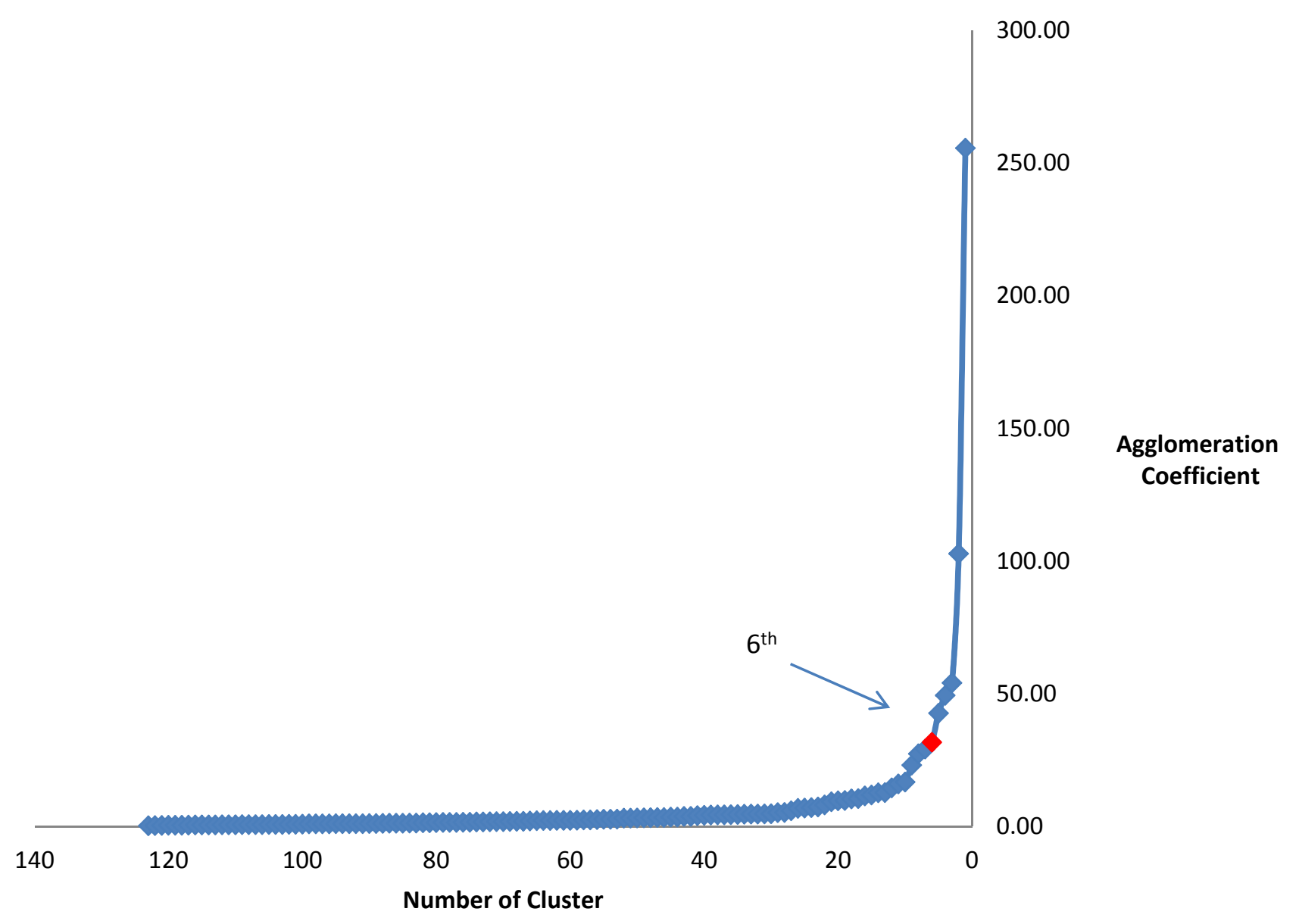

